\title{
Les stratégies d'enseignement clinique qui inspirent et améliorent les connaissances de vos apprenants
}

\section{Par Allan Lai}

\section{Introduction}

$\mathrm{L}$ 'enseignement clinique est devenu une compétence fondamentale dans le domaine des soins infirmiers d'urgence. La demande en soins d'urgence $24 / 24$ na jamais été aussi importante, surtout si l'on prend en compte la pénurie mondiale d'infirmiers/infirmières. Les services d'urgence (SU) embauchent et forment de plus en plus d'infirmiers et infirmières spécialisés pour répondre aux demandes. La technologie évolue à une vitesse qui jamais été observée au cours de l'histoire humaine, ce qui en conséquence entraîne de nouvelles innovations dans le traitement des patients et influence grandement la manière dont les infirmiers et infirmières des services d'urgence pratiquent leur métier. Certains pourraient même dire que cela en va de la responsabilité professionnelle des infirmiers et infirmières d'urgence actuels de former et de soutenir avec succès la prochaine génération d'infirmiers et infirmières d'urgence.

Les pressions auxquelles font face les services d'urgence sont très bien documentées. Quand les infirmiers et infirmières doivent enseigner à d'autres, ils/elles se retrouvent face à de nombreux problèmes, comme un manque d'espace physique pour fournir des soins appropriés, un niveau d'acuité élevé et un va-et-vient constant de nouveaux patients, sans oublier leurs rôles ambigus qu'ils/elles doivent jouer quand ils/elles doivent travailler avec un apprenant. Doivent-ils/elles enseigner ou soigner ? De quelle manière devraient-ils/elles enseigner ? En outre, les pressions associées au temps peuvent donner l'impression que des discussions cliniques plus approfondies ne devraient pas prendre le dessus sur les soins apportés aux patients.

Même si la documentation relative à l'enseignement clinique dans la médecine d'urgence est importante, les recherches de documentation en rapport avec l'enseignement clinique des infirmiers et infirmières d'urgence n'ont donné que peu de résultats. Pour aborder cet écart, cet article va essayer de proposer certaines stratégies d'enseignement efficaces pour les infirmiers et infirmières d'urgence désirant inspirer d'autres et améliorer les connaissances et compétences des apprenants dans les SU.

\section{Une approche structurée}

Les études explorant les caractéristiques des instructeurs cliniques efficaces (telles que définies par des étudiants) suggèrent que ces derniers doivent promouvoir l'autonomie et avoir l'expertise dans des milieux cliniques pertinents, une importante rétroaction et de bonnes compétences de communication (Kelly, 2007; Mailloux, 2006; Tang, Chou \& Chiang, 2005). Pour appliquer ces caractéristiques aux SU, les stratégies suivantes sont présentées en utilisant un moyen mnémonique $A B C D E F G H I$ connu des infirmiers et infirmières d'urgence.
Évaluer l'apprenant, instaurer la confiance, les mettre à jour et $\ll$ diagnostiquer $\gg$ leur apprentissage

Évaluer et reconnaître l'expérience de l'apprenant sont essentiels pour créer une relation professionnelle claire et respectueuse. Étant donné que toutes les conditions médicales, chirurgicale et psychiatriques, à différents degrés, sont présentes dans les SU, une compréhension appropriée de l'expérience préalable de l'apprenant permettra à l'instructeur de mieux identifier les niveaux de compétences et les transitions de l'apprenant (Kramer, 1974; Benner, 1984). Par exemple, une infirmière en formation peut détenir une expérience approfondie dans le domaine des soins intensifs et se sentir confortable avec une séquence rapide d'intubation, mais peut ne pas avoir été exposée par le passé à, ou avoir traité, un saignement pelvien indifférencié. Par conséquent, il est important de mettre à jour les connaissances des apprenants afin d'identifier les besoins et les stratégies d'apprentissage en se basant sur leurs expériences afin qu'ils puissent être mesure de décider comment et ce qu'ils doivent apprendre. En mettant les apprenants dans le siège du conducteur, on les rend responsables de leur apprentissage, tout en conservant les principes de la théorie de l'apprentissage chez les adultes (Kaufman, 2002).

En mettant en place au préalable un certain contexte dans lequel l'apprentissage doit être fait, cela permet à l'infirmier/infirmière d'urgence de « diagnostiquer » les apprenants; cela signifie qu'il faut identifier les types de compétences et d'étapes de transitions qu'ils doivent expérimenter. Un apprenant correctement identifié comme étant bien conscient de ses incompétences, mais pouvant se retrouver face à un choc de transitionnel, nécessitera un mentorat, une rétroaction positive et une normalisation. D'un autre côté, un apprenant « diagnostiqué » comme n'étant pas conscient de ses incompétences, et se trouvant en phase « lune de miel $\gg$, nécessitera un cadre avec des attentes plus réalistes (Kramer, 1974).

\section{Établir des attentes, faciliter, fournir une rétroaction avec un langage clair}

L'infirmier/infirmière d'urgence et l'apprenant doivent établir des attentes dès le début de l'étape clinique. Ils doivent aussi régulièrement et mutuellement évaluer leurs progrès en tout temps. Les attentes doivent être établies en lien avec les compétences de l’apprenant. La majorité du temps, les étudiant(e) $s$ en soins infirmiers d'urgence sont des novices dans leurs approches face aux patients des SU. C'est pour cette raison qu'il est nécessaire de mettre en place des attentes réalistes pour mieux prendre en compte ce niveau novice. Par exemple, une étudiante en soins infirmiers d'urgence peut avoir des connaissances dans la planification et la prestation de soins à des patients souffrant d'anaphylaxie, mais leur rapidité et leurs compétences ne sont 
pas encore entièrement développées. Dans cet exemple, l'infirmier/infirmière d'urgence se doit de partager leur préoccupation et d'aller au-delà du niveau de compétence de l'apprenant, et ils doivent tous deux arriver à une entente mutuelle sur le meilleur moyen d'approcher le patient.

Lorsque l'on applique les pratiques de la théorie d'apprentissage pour les adultes à nos apprenants, les infirmiers et infirmières d'urgence doivent reconnaître que les stagiaires sont autonomes, axés sur la résolution de problèmes, qu'ils doivent apprendre de leurs erreurs et que la véritable conceptualisation de la théorie doit être en rapport avec la pratique (Kaufman 2002). Cette méthode pour dispenser l'éducation est moins associé à « l'enseignement » et plus en rapport avec la facilitation. Par exemple, l'apprenant qui examine un nouveau patient de lui-même en salle de soins peut trouver cela beaucoup plus enrichissant que de l'obliger à examiner un patient spécifique sans le consentement de l'apprenant. En tant que facilitateur, l'infirmier/infirmière d'urgence doit s'assurer que les principales priorités en matière de traitement du patient ne soient pas retardées; telles que les électrocardiogrammes pour les douleurs aigues à la poitrine.

Les patients des SU viennent souvent avec des histoires incomplètes ou sans renseignements accessoires. En permettant à l'apprenant de découvrir cela de lui-même, souvent par le biais de questions posées au patient, et même en commettant quelques erreurs, il sera en mesure de réellement comprendre les nombreuses pratiques associées aux soins infirmiers d'urgence. Dès que l'apprenant semble avoir atteint une certaine phase ou semble stagner dans sa progression, l'infirmier/infirmière d'urgence peut faciliter l'expérience de l'apprenant en posant des questions claires et précises pouvant offrir plusieurs choix de réponses. Ces questions sont généralement ouvertes et sincères et peuvent aider à promouvoir la réflexion de l'apprenant. Une telle question pourrait être : « Pourquoi avez-vous choisi d'appliquer une surveillance continue $\mathrm{SaO} 2$ ? » Évitez de poser des questions directes obligeant l'apprenant à donner une réponse spécifique, comme «Quelles sont les trois conditions nécessaires à une surveillance $\mathrm{SaO} 2$ ? »

L'utilisation d'un langage clair, comme un compte-rendu avec un bon jugement, est une compétence qui permettra d'améliorer la réflexion de l'apprenant. Plaidoyer/demander est une méthode de questionnement claire permettant à l'infirmier/infirmière d'urgence de montrer leur cadre de référence tout en posant des questions sincères au sujet de l'action ou de l'inaction de l'apprenant (Rudolph et al, 2007). Par exemple, l'infirmier/ infirmière d'urgence peut décider de poser une question de cette façon : «J'ai remarqué que tu n’as pas appliqué une sonde $\mathrm{SaO} 2$ lors de ton premier examen. Je pense que cela aurait pu t'aider dans tes prises de décision. Aide-moi à comprendre ton processus de réflexion $\gg$. Cette approche permet à l'infirmier/infirmière d'urgence de révéler leur perspective et d'éviter que l'apprenant ne devine la réponse à donner à l'enseignement, en laissant la voie à une réponse plus ouverte et sincère. Par conséquent, l'apprenant peut donner une réponse pouvant entraîner une discussion clinique plus approfondie, comme : «Je ne pense pas qu'une lecture $\mathrm{SaO} 2$ aurait pu changer ma décision parce que les doigts étaient froids et nous aurions traité la dyspnée quels que soient les résultats de la lecture $\mathrm{SaO} 2 \gg$. Une forte question ouverte peut offrir davantage de points d'apprentissage que plusieurs questions fermées.

Une rétroaction constructive et efficace, dans un service d'urgence doit être respectueux, précis, opportun et être seulement donnée avec le consentement de l'apprenant. Il va sans dire que des erreurs se produiront, mais si elles sont utilisées de façon appropriée, elles peuvent alors devenir de puissants outils d'apprentissage. Lors de la mise en place de l'étape clinique, un(e) infirmier/infirmière d'urgence se doit d'obtenir le consentement de l'apprenant avant de partager la rétroaction. Cette étape permet de créer une relation professionnelle forte, renforçant ainsi la confiance. La rétroaction doit être objective, claire, opportune et être dépourvue de jargon. La rapidité des SU peut parfois faire oublier certaines choses à l'apprenant et, dans certains cas, ce dernier peut ne pas prendre conscience de ses erreurs. Le concept de soi et l'envie d'apprendre sont d'importants facteurs dans la réussite de l'apprenant, et l'infirmier/infirmière d'urgence peut utiliser cela en partageant souvent des rétroactions positives. Une rétroaction positive et constructive devrait permettre à l'apprenant de partager ses points de vue.

\section{Écouter plus, parler moins et donne une certaine indépendance}

Il a été remarqué que les enseignants attendaient, en moyenne, moins d'une seconde pour obtenir une réponse de la part d'un apprenant (Peninciner, 2002). L'infirmier/infirmière d'urgence doit être prêt(e) à accepter un moment de silence avant d'obtenir la réponse de l'apprenant. Préparez-vous à attendre dix secondes ou plus. Cela permettra à l'apprenant d'écouter, d'interpréter et de formuler une réponse. Si vous remarquez que les soins des patients deviennent une priorité et entraîne une limite de temps, alors invitez l'apprenant à conclure rapidement et à revenir quand il sera prêt à partager sa réponse avec vous. En mettant en place cette méthode d'indépendance, cela motive davantage l'apprenant, lui permet d'inclure ses expériences passées à sa réponse et l'oblige à agir plus et à mémoriser moins.

\section{Conclusion}

L'enseignement clinique dans les services d'urgence est à la fois stimulant et gratifiant. En acceptant le caractère unique des SU et en intégrant ces qualités dans les stratégies d'enseignement, il est possible d'offrir aux étudiants en soins infirmiers d'urgence des expériences d'apprentissage enrichissantes. Même si ces stratégies sont fournies ici en format linéaire, comme les cadres d'évaluation d'urgence, il est possible de rester flexible et créatif dans leur intégration.

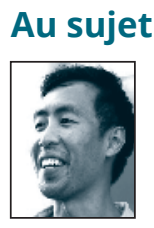

de l'auteur

Allan Lai, BSN, RN, ENC(C), a obtenu son

baccalauréat en sciences infirmières à l'Université

Thompson Rivers (Kamloops, C.B.). Il exerce

actuellement en tant qu'infirmier à l'Hôpital général

de Vancouver et enseigne le programme de spécialité en

soins infirmiers d'urgence à l'Institut de technologie de la ColombieBritannique. Je soutiens également l'équipe de conception nationale EPICC, récemment en tant que membre de l'équipe de projet pour le cours EPICC-Pediatrics à venir. 